\title{
SUPERNOVAE IN FLOCCULENT AND GRAND DESIGN GALAXIES
}

\author{
Marshall L. McCall and Fred H. Schmidt \\ David Dunlap Observatory and University of Toronto
}

\begin{abstract}
The arm structure of supernova host galaxies has been studied in order to ascertain whether or not spiral density waves have an impact on supernovae frequencies. The ensembles of Type I and Type II supernova hosts were found to contain identical fractions of grand design spirals consistent with the representations in control samples chosen without regard to supernovae production. The results suggest that a density wave does not greatly enhance the massive star formation rate per unit luminosity of a galaxy. Instead, star formation in most galaxies may be dominated by stochastic processes.
\end{abstract}

\section{INTRODUCTION}

Grand design spirals are characterized by at least two long and continuous arms symmetrical about the nucleus, highlighted by active regions of star formation. In flocculent galaxies, the arms are short and chaotic, and do not display any bimodal symmetry (see Elmegreen 1981). The two classes of galaxies take on special significance in the context of recent ideas concerning star formation. In flocculent galaxies, star formation appears to be triggered by stochastic processes. For example, the stochastic self-propagating star formation model of Gerola and Seiden (1978) is capable of reproducing the overall appearance of these galaxies. Grand design spirals probably represent systems in which a spiral density wave is present (Roberts, Roberts, and Shu 1975), driven by the action of a companion or an oval distortion (Kormendy and Norman 1979). The coincidence of star formation activity with the arms has led most people to believe that density waves are an additional, and perhaps dominating, trigger of star formation in grand design galaxies.

As yet, it is not known whether or not the star formation rate per unit luminosity or the initial mass function are sensitive to the details of the star formation trigger. Supernovae offer a means of studying these important questions. If density waves enhanced the star formation rate per unit luminosity or preferentially stimulated the formation of massive stars, then grand design spirals would be expected to be more efficient producers of Type II supernovae. The consequence 
would be an over-representation of grand design relative to flocculent galaxies in the ensemble of Type II supernova hosts relative to control samples selected without regard to supernova activity. On the other hand, the frequency of Type I supernovae should be governed by the cumulative history of star formation, and so should be tied fairly closely to galaxy luminosity. Therefore, one might expect that the representation of grand design spirals in the sample of Type I supernova hosts should be close to that in the control samples.

\section{METHOD}

The authors have carried out a study of the arm structure of galaxies which have hosted supernovae in order to assess the impact of density waves on star formation. To distinguish between flocculent and grand design spirals according to a well defined set of rules, the classification system developed by Elmegreen and Elmegreen (1982) was applied. Arm and environmental classifications were derived from inspection of the POSS O plate copies, ESO B films, and SERC J films of the David Dunlap Observatory. Every galaxy which has hosted one or more supernovae up to 1985 May (Kowal 1983, and subsequent IAU telegrams) was studied. All classifiable galaxies with $\log R_{25}$ less than or equal to 0.30 and with a diameter greater than about $1^{\prime}$ mde up the final sample of 115 . Following Elmegreen and Elmegreen (1982), all galaxies having an arm class between 1 and 4 were designated to be flocculent (F), while galaxies showing some degree of bisymmetry, i.e. arm classes 5 through 12, were designated grand design (G).

Two supernova-independent control samples studied by Elmegreen and Elmegreen (1982) were used to assess the importance of selection effects on the statistics for the supernova hosts. The larger, henceforth referred to as the Elmegreen and Elmegreen sample, is comprised of field, binary, and group galaxies in the catalogues of Turner (1976) and Turner and Gott (1976) having an inclination less than $55^{\circ}$ and with $m_{V}$ brighter than 14.0. The second is drawn from the Revised Shapley-Ames Catalogue (Sandage and Tammann 1981).

\section{RESULTS AND CONCLUSIONS}

Of the 115 supernova hosts, $0.76 \pm 0.04$ are grand design spirals. Of the galaxies with typed supernovae, 39 were Type I hosts and and 32 were Type II hosts. Of the Type I hosts, $0.77 \pm 0.07$ are grand design spirals. In comparison, the grand design fraction for the Type II hosts is $0.78 \pm 0.07$. In the Elmegreen and Elmegreen control sample (259 galaxies), $0.69 \pm 0.03$ are grand design spirals. In the Revised Shapley-Ames sample ( 86 galaxies), the grand design fraction is $0.73 \pm 0.05$. The fractions reflect the fact that the grand design spirals in the samples are on average more luminous than the flocculent galaxies.

Overall, the representation of grand design spirals in the sample of supernova hosts is consistent with the representation in the control samples. Since about two-thirds of supernovae are Type I (Branch 1980), this result is reflective of the grand design fraction for the Type I hosts. It is unlikely that the frequency of Type I supernovae varies with arm class in a way which exactly cancels selection effects. 
Table 1. Numbers of Flocculent and Grand Design Galaxies

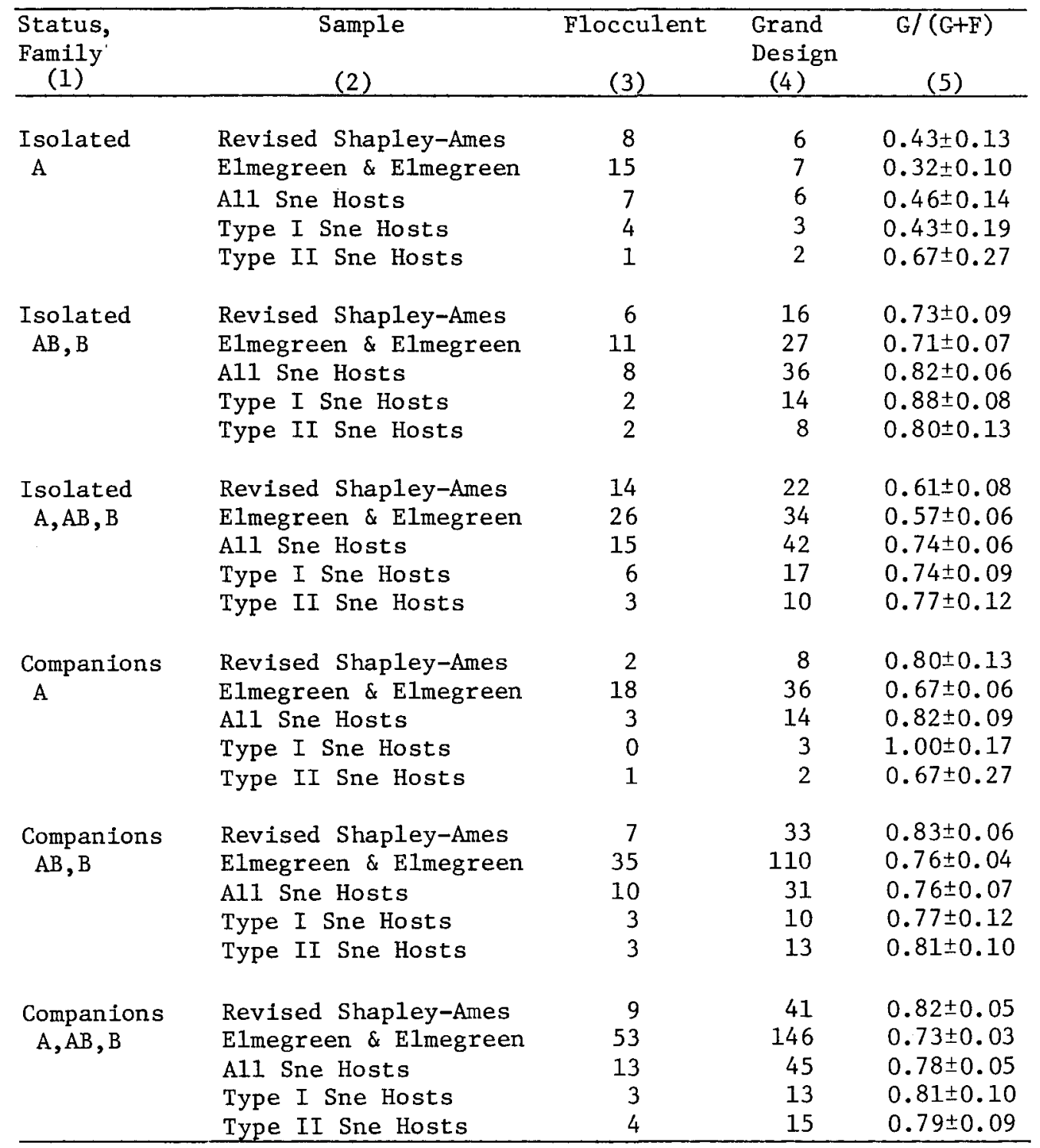

Column 1 categorizes the environment and the family as defined by Elmegreen and Elmegreen (1982) and de Vaucouleurs, de Vaucouleurs, and Corwin (1976), respectively. Column 2 defines the sample. For each category, the two control samples are listed first, followed by samples of supernova hosts chosen on the basis of supernova type. Column 3 lists the number of flocculent galaxies (F), and column 4 the number of grand design galaxies (G). In column 5, the fraction of galaxies which display a grand design is given along with the uncertainty computed from binomial statistics. 
Therefore, the results suggest that the Type I supernova rate is independent of arm class, and that the sample of supernova hosts is unbiased with respect to the control samples. Statistics of subsamples of the supernova hosts, which are summarized in Table 1, support these conclusions. For all environment-family combinations, the representation of grand design spirals in the sample of Type I hosts and overall is consistent with the representation in the controls. In particular, the subsample of isolated non-barred galaxies shows the same drop in the grand design fraction as displayed by the controls.

Now it is possible to assess the significance of the statistics for the grand design spirals in the sample of Type II supernova hosts. In all environment-family groupings, the fraction of grand design spirals is consistent with the fractions for the controls, the entire supernova host ensemble, and, most important, the Type I hosts. Based on the comparison with the Type I sample, the massive star formation rate (per unit luminosity) in the grand design spirals is less than a factor of about 1.7 times that in the flocculents. Certainly, the formation of massive stars in spirals does not hinge upon the existence of a density wave. The results suggest that star formation in most galaxies is dominated by stochastic processes.

\section{REFERENCES}

Branch, D. 1980, in Supernova Spectra, eds. Meyerott, R. E., and Gillespie, G. H. (New York: AIP), p. 39.

de Vaucouleurs, G., de Vaucouleurs, A., and Corwin, H. G., Jr. 1976, Second Reference Catalogue of Bright Galaxies (Austin: University of Texas Press).

Elmegreen, D. M. 1981, Ap. J. Suppl., 47, 229.

Elmegreen, D. M., and Elmegreen, B. G. 1982, M.N.R.A.S., 201, 1021.

Gerola, H., and Seiden, P. E. 1978, Ap. J., 223, 129.

Kormendy, J., and Norman, C. A. 1979, Ap. J., 233, 539.

Kowal, C. T. 1983 (October), unpublished.

Roberts, W. W., Roberts, M. S., and Shu, F. H. 1975, Ap. J., 196, 381.

Sandage, A., and Tammann, G. A. 1981, A Revised Shapley-Ames Catalog of Bright Galaxies (Washington: Carnegie Institution).

Turner, E. L. 1976, Ap. J., 208, 20.

Turner, E. L., and Gott, J. R. III 1976, Ap. J. Suppl., 32, 409. 